\title{
Strong violations of Bell-type inequalities for Werner-like states
}

\author{
Christoph F. Wildfeuer ${ }^{1, *}$ and Jonathan P. Dowling ${ }^{1}$ \\ ${ }^{1}$ Hearne Institute for Theoretical Physics, Department of Physics and Astronomy, \\ Louisiana State University, Baton Rouge, Louisiana 70803, USA
}

\begin{abstract}
We investigate the violation of Bell-type inequalities for two-qubit Werner-like states parametrized by the positive parameter $0 \leq p \leq 1$. We use an unbalanced homodyne detection scheme to obtain the quantum mechanical probabilities. A violation of the Bell-Wigner and Janssens inequalities is obtained for a large range of the parameter $p$. The range given by these inequalities is greater than the one given by the Clauser-Horne inequality. The range in which a violation is attained actually coincides with the range where the Werner-like states are known to be nonseparable, i.e., for $p>1 / 3$. However, the improvement over the Clauser-Horne inequality is achieved at the price of restricting the class of possible local hidden variable theories.
\end{abstract}

PACS numbers: 03.65.Ud, 03.65.Wj, 03.67.Mn, 42.50.Xa

\section{INTRODUCTION}

Since the seminal paper of Einstein, Podolsky, and Rosen [1] on the completeness and physical reality of quantum mechanics, a great deal of research has been devoted to clarify the relation between nonseparable quantum states and nonlocal correlations. This relation, as it turns out, is far from trivial. For an overview see, e.g., Ref. 2].

If a quantum state is nonseparable, i.e, cannot be factored in some product of its subsystems, it is called entangled. Although a state may be entangled, it need not necessarily manifest nonlocal correlations. It was Bell that suggested an operational criterion to test the predictions of local hidden variable (LHV) theories against quantum mechanical predictions. The violation of a Bell inequality by a specific quantum state is then an indication that the state is able to exhibit nonlocal correlations. Almost all current experimental tests of nonlocality are based on the Clauser-Horne $(\mathrm{CH})$ inequality 3], the Clauser-Horne-Shimony-Holt (CHSH) inequality [4], and a few on the Wigner inequality [5].

It is known that for any entangled pure state of any number of quantum systems one may violate a generalized Bell inequality [6, 7]. An extension of this statement for mixed entangled states has not been found. Furthermore, Werner [8] provided in 1989 an example of nonseparable mixed states that do not violate the $\mathrm{CHSH}$ inequality. He then constructed a LHV model which replicated the properties of his mixed quantum states, which have since become known as Werner states. This demonstrated that one cannot always violate Bell inequalities with any mixed entangled state. Two-qubit Werner states are parametrized by the positive parameter $0 \leq p \leq 1$ and can be expressed as follows:

$$
\rho_{p}^{\mathrm{W}}=p|\Psi\rangle\langle\Psi|+\frac{(1-p)}{4} \mathbb{I} .
$$

*Electronic address: wildfeuer@phys.lsu.edu
The Werner states are a mixture of an entangled pure state $|\Psi\rangle$ with noise. Werner showed that these states admit a LHV model for projective measurements for $1 / 3<p \leq 1 / 2$ and violate the $\mathrm{CHSH}$ inequality for $p>1 / \sqrt{2}$. It is known that these states are separable if and only if $p \leq 1 / 3$ [8]. A number of authors have further investigated the nonlocal properties of Werner states 9, 10, 11, 12, 13]. Recently, Acín et al. [10] have extended the range for which a LHV model for Werner states can be constructed. It is not known whether Werner states admit a LHV model for projective measurements in the region $0.66<p \leq 1 / \sqrt{2}$, or if there is a Bell inequality that may be violated, which refers to the gap in Fig. 6. Inspired by this open problem it is interesting to investigate the amount of violation obtained for various Bell-type inequalities with Werner states or states of similar structure than Werner states. It appears that the amount of nonlocality obtained in an experiment depends on the specific Bell-type inequality tested and the measurement carried out.

A very powerful geometrical interpretation of Bell-type inequalities was introduced by Pitowsky in 1986, where they are referred to as correlation polytopes [14]. He showed that the probabilities in a Bell-type inequality can be considered to be a vector in a convex polytope. One can think of the points contained within this polytope as each representing a set of measurement probabilities satisfying a Bell-type inequality, which is represented by the bounding planes of the polytope itself. This enables one to use tools from convex geometry to construct other Bell-type inequalities relevant for other quantum tests of nonlocality [15]. Recently Janssens et al. [16] generated in this way Bell-type inequalities in the correlation polytope for six joint probabilities which may be used to test local realism in physical experiments. The structure of these inequalities is very similar to the BellWigner inequality.

The article is organized as follows: We start our investigation with an inequality from the well-known ClauserHorne polytope. We then investigate the violation of an inequality from the lesser well-known Bell-Wigner polytope which contains three joint probabilities. Finally we 
present an analysis of Janssens' Bell-type inequalities in the correlation polytope for six joint probabilities. The single and joint probabilities for the Bell tests are calculated explicitly for an unbalanced homodyne detection scheme, where the two detectors are assumed to be spacelike separated. We consider the two-qubit Werner state from Eq. (1) for all Bell tests presented. Note that $\mathbb{I}$ is the identity operator in the two-qubit space. For the pure entangled state $|\Psi\rangle$ we consider the one-photon entangled state

$$
|\Psi\rangle=\frac{1}{\sqrt{2}}\left(|1\rangle_{a}|0\rangle_{b}-|0\rangle_{a}|1\rangle_{b}\right),
$$

where the labels $a$ and $b$ refer to the two path-entangled modes accessible to Alice and Bob, respectively.

There has been a long debate about the actual nature of entanglement for the one-photon entangled state because it contains only one particle. A few authors argued that at least two particles are required to make a proper entangled state. Some arguments can be found in Refs. [17, 18] and citations therein. Van Enk has since pointed out that the two field modes themselves should be considered entangled rather than the individual photons - an approach in which the dispute appears to be settled [18].

Although this state can now clearly be called entangled until very recently it has been questioned whether or not a single photon entangled state exhibit nonlocal correlations. Since the very thorough investigation by Dunningham and Vedral [19], there can be no doubt anymore that single particles in a delocalized state can exhibit nonlocal correlations. The authors furthermore point out that "we must not view nonlocality as pertaining to particles themselves, but see it instead as a property of quantum fields whose significance is, therefore, more fundamental than that of particles." Some recent experiments are also in agreement of the nonlocal nature of the one-photon entangled state discussed in this article [20].

Note that many authors use the above notation to refer to spin up and down, i.e., $|1\rangle=|\uparrow\rangle$ and $|0\rangle=|\downarrow\rangle$, which refers to a different physical system and hence leads to other correlation functions, i.e., probability distributions. The results obtained in Refs. [9, 10, 11, 12, 13] are obtained for the spin-1/2 singlet and the usual traceless spin projection operators. Therefore they do not need to coincide with our results obtained for the one-photon entangled state. Note also the projection operators given in Eqs. (3), and (4) are not traceless. For the sake of clarity we emphasize that we refer to Werner-like states in our article. We leave the term Werner states for the original approach in which the maximally entangled pure two-qubit state is considered to be the spin- $1 / 2$ singlet and the measurement operators are the spin projectors.

\section{UNBALANCED HOMODYNE DETECTION SCHEME}

We calculate the quantum mechanical probabilities for our Bell test for an unbalanced homodyne detection scheme as displayed in Fig. 11. State reconstruction with

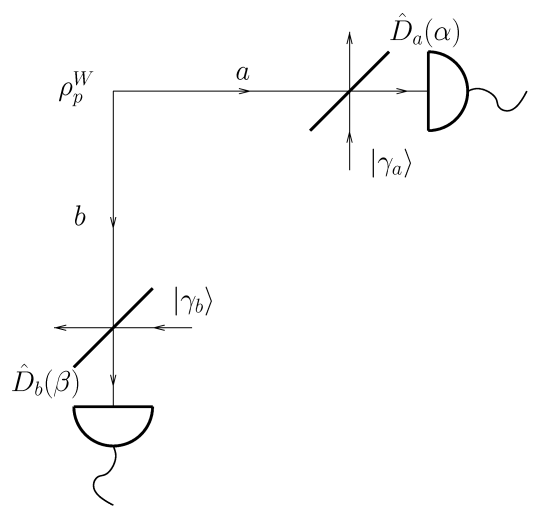

FIG. 1: Unbalanced homodyne detection scheme for a Bell experiment with Werner-like states. Here $\rho_{p}^{\mathrm{W}}=p|\Psi\rangle\langle\Psi|+$ $\frac{(1-p)}{4} \mathbb{I}$ with $|\Psi\rangle=\frac{1}{\sqrt{2}}\left(|1\rangle_{a}|0\rangle_{b}-|0\rangle_{a}|1\rangle_{b}\right)$ and $a$ and $b$ label the modes. Alice and Bob mix mode matched local oscillators of amplitude $\gamma_{a}$ and $\gamma_{b}$, respectively, with their signal modes, where $\alpha=\gamma_{a} \sqrt{1-T}$ and $\beta=\gamma_{b} \sqrt{1-T}$ are the arguments of the displacement operator $\hat{D}$ defined in the text. The symbol $T$ denotes the transmittivity of the beam splitters.

an unbalanced homodyne detection scheme has been investigated in detail by Wallentowitz and Vogel [21]. The authors showed that the $Q$ function can be measured directly in an unbalanced homodyne detection scheme with a perfect detector by recording the probability of zero counts as a function of the complex amplitude of the local oscillator. The photon detectors are considered to be simple on-off detectors, i.e., they cannot resolve the photon number. The local projective measurements are described by $\hat{Q}(\alpha)+\hat{P}(\alpha)=\hat{\mathbf{1}}$, with

$$
\begin{aligned}
& \hat{Q}(\alpha)=\hat{D}(\alpha)|0\rangle\langle 0| \hat{D}^{\dagger}(\alpha), \\
& \hat{P}(\alpha)=\hat{D}(\alpha) \sum_{n=1}^{\infty}|n\rangle\langle n| \hat{D}^{\dagger}(\alpha) .
\end{aligned}
$$

The displacement operator is defined by

$$
\hat{D}(\alpha)=\exp \left(-\frac{1}{2}|\alpha|^{2}\right) \exp \left(\alpha \hat{a}^{\dagger}\right) \exp \left(-\alpha^{*} \hat{a}\right),
$$

22. The expectation value of $\hat{Q}(\alpha)$ tells us the probability that no photons are present, depending on the phase and amplitude of the local oscillator. The expectation value of $\hat{P}(\alpha)$ gives the probability of counting one or more photons. We obtain a binary result by assigning a one to a detector click and a zero otherwise. The corresponding measurement operators for a correlated measurement of the displaced vacuum can be written as 
$\hat{Q}_{a}(\alpha) \otimes \hat{Q}_{b}(\beta)$. The joint probability for the Werner-like state is calculated from

$$
Q_{a b}(\alpha, \beta)=\operatorname{Tr}\left(|\alpha\rangle_{a}\langle\alpha|\otimes| \beta\rangle_{b}\langle\beta| \rho_{p}^{\mathrm{W}}\right),
$$

and we obtain

$$
\begin{aligned}
& Q_{a b}(\alpha, \beta)=\frac{p}{2} e^{-|\alpha|^{2}-|\beta|^{2}}\left(|\alpha-\beta|^{2}\right) \\
& \quad+\frac{1-p}{4} e^{-|\alpha|^{2}-|\beta|^{2}}\left(1+|\alpha|^{2}+|\beta|^{2}+|\alpha|^{2}|\beta|^{2}\right) .
\end{aligned}
$$

The single-count probability for Alice's measurement is given by

$$
Q_{a}(\alpha)=\operatorname{Tr}\left(|\alpha\rangle_{a}\langle\alpha| \otimes \hat{\mathbf{1}}_{b} \rho_{p}^{\mathrm{W}}\right)=\frac{1}{2} e^{-|\alpha|^{2}}\left(|\alpha|^{2}+1\right),
$$

and the corresponding probability for Bob's measurement of $Q_{b}(\beta)$ is

$$
Q_{b}(\beta)=\operatorname{Tr}\left(\hat{\mathbf{1}}_{a} \otimes|\beta\rangle_{b}\langle\beta| \rho_{p}^{\mathrm{W}}\right)=\frac{1}{2} e^{-|\beta|^{2}}\left(|\beta|^{2}+1\right) .
$$

Alice and Bob obtain the same probability distribution for their independent measurements. Note also that the joint probabilities in Eq. (6) are symmetric in the two arguments, i.e., $Q(\alpha, \beta)=Q(\beta, \alpha)$. We may therefore drop the subindices on the $Q$ functions for what follows. We can assume that the first argument for the joint probabilities $Q(\alpha, \beta)$ is Alice's local oscillator ( $\mathrm{LO})$ setting and the second argument belongs to Bob's LO. The single-count probabilities can essentially be obtained from a run of the experiment at either Alice's or Bob's laboratory. With the above single and joint probabilities, we can test if any of the Bell-type inequalities are violated.

\section{VIOLATION OF CLAUSER-HORNE AND WIGNER INEQUALITIES}

We investigate the violation of the well-known $\mathrm{CH}$ inequality [3], given by $-1 \leq \mathcal{I}_{C H} \leq 0$, where $\mathcal{I}_{C H}$ is defined as follows:

$$
\begin{aligned}
\mathcal{I}_{C H}= & Q(\alpha, \beta)-Q\left(\alpha, \beta^{\prime}\right)+Q\left(\alpha^{\prime}, \beta\right)+Q\left(\alpha^{\prime}, \beta^{\prime}\right) \\
& -Q\left(\alpha^{\prime}\right)-Q(\beta) .
\end{aligned}
$$

A minimization method for the parameters $\alpha, \alpha^{\prime}, \beta$, and $\beta^{\prime}$ results in a violation as a function of $p$ as displayed in Fig. 2. We attain a violation for any $p>0.75$. If we consider the Werner state in the spin- $1 / 2$ basis and decide to make a spin correlation measurement instead, we can violate the $\mathrm{CH}$ inequality for any $p>1 / \sqrt{2} \approx 0.71$, which is well known [8].

The Werner-like state in the Fock basis together with a measurement that projects onto coherent states, as given by Eq. (3), obviously leads to a different result for the range of violation than the Werner state in the spin basis and a spin correlation measurement. Note also that the operators given in Eqs. (3), and (4) are not traceless.

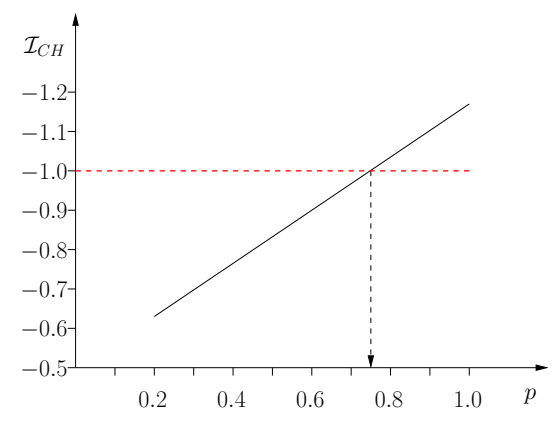

FIG. 2: (Color online) Violation of the $\mathrm{CH}$ inequality $-1 \leq$ $\mathcal{I}_{C H} \leq 0$ with $\mathcal{I}_{C H}$ defined in Eq. (9) as a function of the mixing parameter $p$. The horizontal dashed line marks the classical constraint. We obtain a violation for $p>0.75$.

Therefore the known bounds for traceless operators as derived in Ref. [10] cannot be directly applied.

Next we consider one of the inequalities from the BellWigner polytope [23] given by $W_{1} \leq 1$, where

$$
\begin{aligned}
W_{1}= & Q(\alpha)+Q(\beta)+Q(\gamma) \\
& -Q(\alpha, \beta)-Q(\alpha, \gamma)-Q(\beta, \gamma) .
\end{aligned}
$$

Note that original Bells' inequality 24] cannot be applied to noisy entangled states such as the $\mathrm{CH}$ and $\mathrm{CHSH}$ can. This is due to the fact that Bell's approach works only for perfect anticorrelated pure states. This assumption is no longer fulfilled for mixed entangled states where the correlations may not be perfect anymore. In a recent paper by Pitowsky it is shown that Bell's original approach can be extended to mixed entangled states if the states are not too noisy [25]. Pitowsky's extension of Bell's inequality to mixed entangled states also works for the Bell inequalities we are going to consider in this and the next section. Pitowsky also considers two limiting cases which make further assumptions about the distribution of the local hidden variables. The inequalities we are considering are valid for the assumption of zero average symmetry breaking [25]. We note that the extra assumptions on the distribution of the local hidden variables restrict our approach to a specific class of local hidden variable models. However, the fair sampling assumption, which is commonly assumed in all experiments, already rules out a large class of local hidden variable models.

A maximization method for the complex parameters $\alpha, \beta$, and $\gamma$ results in a violation as a function of $p$ as displayed in Fig. 3 From Fig. 3 we see that the range $p$ for which the value $W_{1}$ violates the classical constraint is very large. A further analysis shows that one may numerically approach the separability-nonseparability border at $p=1 / 3$ with a high precision. We see that the above test of the Bell-Wigner inequality shows a violation for the two-qubit Werner-like state for the entire nonseparability range $p>1 / 3$. The Bell-Wigner inequality is obviously a stronger inequality than the Clauser-Horne inequality, in the sense that it shows a larger range of violation for the Werner-like state. 


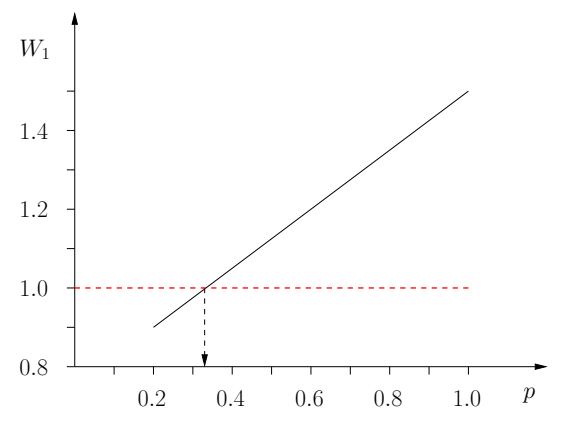

FIG. 3: (Color online) Violation of the Bell-Wigner inequality $W_{1} \leq 1$ with $W_{1}$ defined in Eq. (10) as a function of the mixing parameter $p$. The horizontal dashed line marks the classical constraint. We obtain a violation for $p>1 / 3$.

\section{NEW BELL TESTS}

We finally consider Bell-type inequalities which, to our knowledge, are not currently used in Bell tests. These Bell-type inequalities belong to the correlation polytope for six joint probabilities, which have been constructed by Janssens et al. [16]. We display them first in terms of classical single and joint probabilities, where the probability of a single random event $A_{i}$ is defined by $p_{i}=P\left(A_{i}\right)$ and the joint probability of a pair of random events $A_{i}$ and $A_{j}$ is denoted by $p_{i j}=P\left(A_{i} \cap A_{j}\right)$ :

$$
\begin{gathered}
0 \leq p_{i}+p_{j}+p_{i j}-p_{i k}-p_{i \ell}-p_{j \ell}-p_{j k}+p_{k \ell}, \\
p_{i}+p_{j}+p_{k}+p_{\ell}-p_{i j}-p_{i k}-p_{i \ell}-p_{j k}-p_{j \ell}-p_{k \ell} \leq 1 \\
2 p_{i}+2 p_{j}+2 p_{k}+2 p_{\ell}-p_{i j}-p_{i k}-p_{i \ell}-p_{j k}-p_{j \ell}-p_{k \ell} \leq 3, \\
0 \leq p_{i}-p_{i j}-p_{i k}-p_{i \ell}+p_{j k}+p_{j \ell}+p_{k \ell}, \\
p_{i}+p_{j}+p_{k}-2 p_{\ell}-p_{i j}-p_{i k}+p_{i \ell}-p_{j k}+p_{j \ell}+p_{k \ell} \leq 1
\end{gathered}
$$

for any different $i, j, k, \ell$. We investigate the amount of violation of the inequalities in Eqs. (11)-(15) for the simple on-off detection scheme, with the probabilities given by Eqs. (6), (7), and (8). The probabilities for the inequality (11) are then replaced by

$$
\begin{array}{r}
J_{1}=Q(\alpha)+Q(\beta)+Q(\alpha, \beta)-Q(\alpha, \gamma)-Q(\alpha, \delta) \\
-Q(\beta, \delta)-Q(\beta, \gamma)+Q(\gamma, \delta),
\end{array}
$$

so that the inequality is expressed by $0 \leq J_{1}$. We display the results for the minimization procedure in Fig. 4. We obtain a violation for $p>0.4$ which is linearly increasing with $p$ until it reaches the maximum for $p=1$. For the violation of the pure one-photon entangled state compare with Ref. [26] also.

As another example of the violations obtained in this correlation polytope, we investigate the inequality (13).

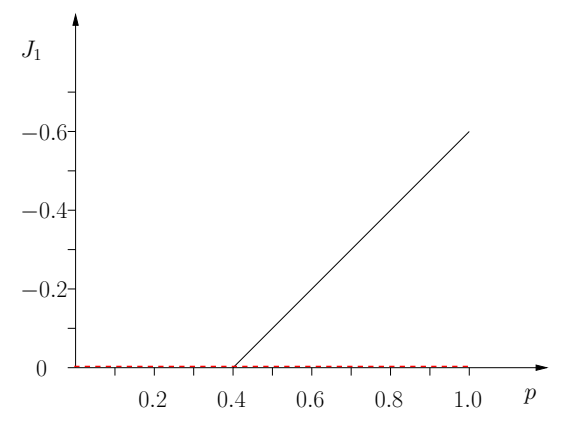

FIG. 4: (Color online) Violation of the Bell-type inequality $0 \leq J_{1}$, where $J_{1}$ is defined in Eq. (16), as a function of $p$. The horizontal dashed line marks the classical constraint. We obtain a violation for $p>0.4$.

This can be rewritten in terms of the local oscillator amplitudes as well, where we introduce the quantity $J_{3}$ by

$$
\begin{aligned}
J_{3}= & 2 Q(\alpha)+2 Q(\beta)+2 Q(\gamma)+2 Q(\delta)-Q(\alpha, \beta) \\
& -Q(\alpha, \gamma)-Q(\alpha, \delta)-Q(\beta, \gamma)-Q(\beta, \delta)-Q(\gamma, \delta),
\end{aligned}
$$

to write the inequality in the form $J_{3} \leq 3$. In Fig. 5 we show again the violation as a function of $p$. The range

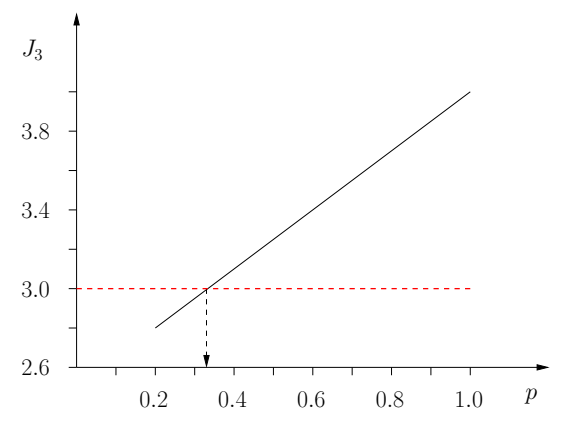

FIG. 5: (Color online) Violation of the Bell-type inequality $J_{3} \leq 3$, where $J_{3}$ is defined in Eq. (17), as a function of $p$. The horizontal dashed line marks the classical constraint. We obtain a violation for $p>1 / 3$.

of violation is exactly the same as for the Bell-Wigner inequality, where we obtain a violation for any $p>1 / 3$.

\section{CONCLUSION}

We conclude from our results that Bell-type inequalities exist, which are violated for the entire range in which Werner-like states are nonseparable, i.e., entangled. In the unbalanced homodyne detection scheme, we showed that the Werner-like states violate one of the well established Bell-Wigner inequalities for the entire range $p>1 / 3$. We also presented Bell-type inequalities in the correlation polytope of six joint probabilities that have not been investigated so far in the context of experiments 
on local realism. Some of these inequalities also give a violation for the full range where the Werner-like states are entangled. We may therefore add to the overview given in Acín et al.'s publication [10] our result and obtain Fig. 6. Our result also shows that Bell-type inequalities

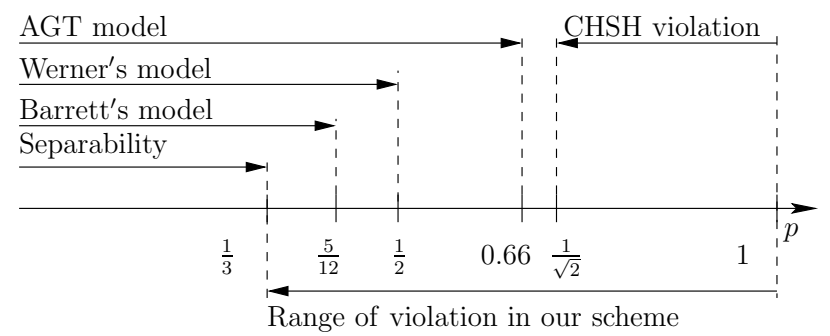

FIG. 6: Overview of the current status on the violation of Bell-type inequalities in different models by two-qubit Werner states $\rho_{p}^{\mathrm{W}}$ as a function of $p$. The models refer to the following publications: Barrett [11], Werner 8], Acín, Gisin, and Toner (AGT) [10].

other than the $\mathrm{CH}$ or $\mathrm{CHSH}$ are less sensitive when noise is added to the pure entangled state $|\Psi\rangle$. Several authors, see, e.g., Refs. [10, 27] and the references therein, have investigated the robustness of Bell tests against noise. The inequalities from the Bell-Wigner and Janssens correlation polytope are obviously advantageous for Bell tests under the influence of noise. It should be noted, however, that for our specific measurement scheme, this advantage does not seem to be related to the dimensionality of the correlation polytope, since the Bell-Wigner inequality with three joint probabilities shows the same violation as the Janssens inequality with six joint probabilities. In fact, this noise-resistant feature seems much more closely tied to the method used to construct the joint probabilities. The Bell-Wigner inequalities allow Alice, for example, to perform a measurement with the LO settings $\alpha$ and $\beta$ and Bob with $\beta$ and $\gamma$. We see that one setting, here $\beta$, is assigned once to Alice's laboratory and in another run of the experiment to Bob's laboratory. Pitowsky showed that a local hidden variable model can be constructed for this combination of events, so that the violation of the Bell-Wigner inequality is a true indication for the violation of the locality assumption. However our approach is only valid for a restricted class of local hidden variable models in which zero average symmetry breaking is assumed 25]. Note that Janssens' inequalities similarly combine the measurements which Alice and Bob may perform, merely taking more combinations into account. This is different from the structure in the $\mathrm{CH}$ inequality. Here Alice accesses the LO settings $\alpha$ and $\alpha^{\prime}$ while Bob can perform a measurement with $\beta$ and $\beta^{\prime}$. Alice and Bob always have distinct LO settings in their laboratories. We point out that our example demonstrates a way in which other Bell-type inequalities can be valuable.

\section{Acknowledgments}

C.F.W. and J.P.D. acknowledge the ARO and the DTO for support. We thank H. Lee, S.J. Olson, W. Plick, M.M. Wilde, K. Jacobs, N. Sauer, R. Kretschmer, M.P. Seevinck, J. Bergou, and B. He for very helpful comments.
[1] A. Einstein, B. Podolsky, and N. Rosen, Phys. Rev. 47, 777 (1935).

[2] M. Genovese, Phys. Rep. 413, 319 (2005).

[3] J. F. Clauser and M. A. Horne, Phys. Rev. D 10, 526 (1974).

[4] J. F. Clauser, M. A. Horne, A. Shimony, and R. A. Holt, Phys. Rev. Lett. 23, 880 (1969).

[5] E. P. Wigner, Am. J. Phys. 38, 1005 (1970).

[6] N. Gisin and A. Peres, Phys. Lett. A 162, 15 (1992).

[7] S. Popescu and D. Rohrlich, Phys. Lett. A 166, 293 (1992).

[8] R. F. Werner, Phys. Rev. A 40, 4277 (1989).

[9] N. Gisin, e-print arXiv: quant-ph/0702021v2.

[10] A. Acín, N. Gisin, and B. Toner, Phys. Rev. A 73, 062105 (2006).

[11] J. Barrett, Phys. Rev. A 65, 042302 (2002).

[12] S. Popescu, Phys. Rev. Lett. 74, 2619 (1995).

[13] S. Teufel, K. Berndl, D. Dürr, S. Goldstein, and N. Zanghi, Phys. Rev. A 56, 1217 (1997).

[14] I. Pitowsky, J. Math. Phys. 27, 1556 (1986).

[15] S. Filipp, and K. Svozil, e-print arXiv: quant-ph/0105083v1.

[16] S. Janssens, B. De Baets, and H. De Meyer, Fuzzy Sets Syst. 148, 263 (2004).
[17] S. J. van Enk, Phys. Rev. A 72, 064306 (2005).

[18] S. J. van Enk, Phys. Rev. A 74, 026302 (2006).

[19] J. Dunningham and V. Vedral, Phys. Rev. Lett. 99, 180404 (2007).

[20] B. Hessmo, P. Usachev, H. Heydari, and G. Björk, Phys. Rev. Lett. 92, 180401 (2004); S. A. Babichev, J. Appel, and A. I. Lvovsky, ibid. 92, 193601 (2004); M. D'Angelo, A. Zavatta, V. Parigi, and M. Bellini, Phys. Rev. A 74, 052114 (2006).

[21] S. Wallentowitz and W. Vogel, Phys. Rev. A 53, 4528 (1996).

[22] C. C. Gerry and P. L. Knight, Introductory Quantum Optics (Cambridge University Press, Cambridge, 2005).

[23] I. Pitowsky, Quantum Probability-Quantum Logic, Lecture Notes in Physics No. 321 (Springer, Berlin, 1989).

[24] J. S. Bell, Physics (Long Island City, N.Y.) 1, 195 (1964).

[25] I. Pitowsky, J. Math. Phys. 49, 012101 (2008).

[26] C. F. Wildfeuer, A. P. Lund, and J. P. Dowling, Phys. Rev. A 76, 052101 (2007).

[27] D. Collins, N. Gisin, N. Linden, S. Massar, and S. Popescu, Phys. Rev. Lett. 88, 040404 (2002); A. Acín, T. Durt, N. Gisin, and J. I. Latorre, Phys. Rev. A 65, 052325 (2002). 